\title{
Octree-Based Production of Near Net Shape Components
}

\author{
H. Medellín, J. R. Corney, J. B. C. Davies, T. Lim, and J. M. Ritchie
}

\begin{abstract}
Near net shape (NNS) manufacturing refers to the production of products that require a finishing operation of some kind. NNS manufacturing is important because it enables a significant reduction in: machining work, raw material usage, production time, and energy consumption. This paper presents an integrated system for the production of near net shape components based on the Octree decomposition of 3-D models. The Octree representation is used to automatically decompose and approximate the 3-D models, and to generate the robot instructions required to create assemblies of blocks secured by adhesive. Not only is the system capable of producing shapes of variable precision and complexity (including overhanging or reentrant shapes) from a variety of materials, but it also requires no production tooling (e.g., molds, dies, jigs, or fixtures). This paper details how a number of well-known Octree algorithms for subdivision, neighbor findings, and tree traversal have been modified to support this novel application. This paper ends by reporting the construction of two mechanical components in the prototype cell, and discussing the overall feasibility of the system.
\end{abstract}

Note to Practitioners-Traditional NNS manufacturing systems (e.g., forging, injection molding, and casting) require the design and manufacture of production tooling such as molds, dies, jigs, or fixtures to produce NNS components. Therefore, the production time of NNS parts is significantly affected by the need for production tooling. A new method to manufacture NNS parts directly from CAD information is presented in this paper. The proposed system is based on the automatic decomposition of 3-D models into a hierarchy of cubes of different sizes, which are assembled automatically using an industrial robot. There are several technical advantages of the proposed system over existing methods: it does not require the design and manufacture of production tooling; it can build parts from any rigid material (i.e., wood, plastic, ceramic, metal alloy, etc.); the build rate is extremely quick compared to say laminar manufacturing methods or direct CNC machining. However, the system is also significant because it demonstrates an approach to automated NNS manufacture that can be implemented using standard hardware (i.e., a pick and place robot and conveyor feeder) and can be easily scaled to macro or microapplications. The production of several test components has proved that the proposed system is practical as described. Future work considers the automatic postprocessing of NNS components to produce net shape parts automatically, and the investigation of alternatives to adhesives bonding of the assembly.

Index Terms-Near net shape (NNS), NNS component, octree assembly cell, octree decomposition, octree model, RPNNS system.

\section{INTRODUCTION}

$\mathbf{N}$ EAR NET SHAPE (NNS) manufacturing refers to the fabrication of products which can be used directly as a part requiring a finishing operation of some kind. NNS manufacturing is important because it enables a significant reduction in: machining work, raw material usage, production time, and energy consumption [1]. Traditional NNS manufacturing processes include forging, injection molding, and casting. The main characteristic of these processes is that shapeless raw material is formed by a tool (i.e., molds, dies, and cast) with a cavity into the shape of the desired part. NNS is particularly important when high-cost materials are used or when high-volume production of low-cost materials is required. The traditional development cycle of NNS manufactured parts consist of part design, mold (or die) design, mold (or die) manufacturing, and NNS manufacturing [2]. So, the cost, quality, and production time of NNS parts are dominated by that of molds, or dies, which in turn are directly linked to the complexity of part's geometry.

Even though the NNS processes may have a great impact in the production of components, NNS manufacturing systems have been less studied and much of the research works reported consider the analysis and improvement of traditional NNS processes. The following paragraphs review a number of research projects related to $\mathrm{CAD} / \mathrm{CAM}$ support of NNS manufacturing systems. This work can be broadly classified into two categories: 1) development of CAD/CAM tools to support existing NNS technologies and 2) the creation of entirely new NNS manufacturing processes. Considering each of these in turn.

\section{A. CAD/CAM Tools for Existing Processes}

Several researchers have considered the long established process of forging: work on simulation of incremental forging of near net shaped has been presented in [3]; an analysis and the results of some experiments in NNS forging of spur gear forms were presented in [4]; a prototype forming system consisting of four heating modules, a robot for billet handling and a high pressure die casting machine has been reported in [5] and 
assessed for the production of lightweight NNS components; an experimental investigation made into the quasi-static progressive incremental closed-die forging of crown gears forms starting initially from both solid and hollow circular cylindrical specimens were presented in [6]. Similar work on virtual prototyping approaches to support casting processes can also be found; for example, the geometric moldability analysis of NNS manufactured parts was reported in [2].

\section{B. CAD/CAM Support of New NNS Processes}

Much of the motivation for the creation of new processes has been driven by the demands of new materials. For example, there is a distinct focus of NNS research in the area of ceramic materials manufacture. Much of this deals with development of mechanical processes rather than geometric computation. For example, [7] reports the use of explosive and electromagnetic high energy rate compacting forming "powder-in-tube" techniques for NNS manufacturing of axisymmetric silver/superconducting ceramic; [8] describes a new NNS forming process for alumina; [9] details a novel NNS manufacturing method for polymer derived ceramics; [10] presents a study of gelcasting technique for ceramics and finally, [11] reports a new NNS method of fabricating diphasic ceramic microstructures with controlled phase dimensionality and anisotropy. A review of NNS manufacturing processes for ceramics was presented in [12].

However, NNS developments are also motivated by the availability of new computational tools (e.g., robust geometric modeling) or the availability of processes (e.g., cheap laser source). The emergence of main stream layered manufacturing systems has been an outstanding example of this trend. However, in the area of NNS manufacturing, these new tools have enabled the investigation of many novel new process. For example, see the following.

A hybrid system that combines the strengths of selective laser sintering (SLS) and hot isostatic pressing (HIP) for NNS fabrication is presented in [13]. The use of plasma spraying for producing freestanding NNS components of metallic, ceramic and layered composites with alternate ceramic and metallic layer systems was studied in [14]. The application of bulge-forming processes to NNS manufacture is studied in [15]. The use of the twin-strand technique of electromagnetic casting in the aluminium industry for the casting of NNS products was investigated in [16]. The NNS manufacturing of components using direct laser fabrication technology was presented in [17]. The application of laser-aided technologies to the NNS forming of a high-strength titanium alloy was presented in [18]. More recently, a system for developing full-scale prototypes from smallscale physical models was presented in [19].

In summary, NNS manufacturing is an important step in many manufacturing processes for several type of material and the availability of geometric representations and automated systems is creating many opportunities for new processes. However, only a limited amount of NNS manufacturing research has been reported in the literature and this has been focused in three areas.

1) The study of traditional NNS processes.

2) The NNS manufacture of ceramic components.

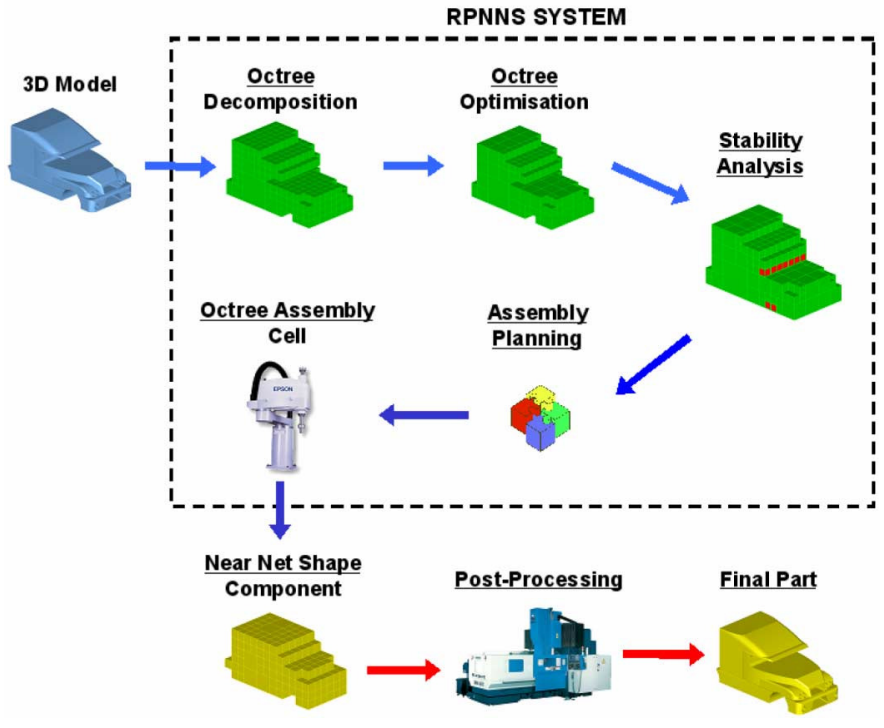

Fig. 1. Overview of the proposed RPNNS system.

3) New NNS manufacturing methods using existing processes such as plasma spraying and direct laser fabrication.

In this context, this paper reports work in the third group and is concerned with the development of a novel technique for the rapid production of NNS models (named RPNNS). The proposed technique uses traditional manufacturing processes and extends established approaches of geometric representation and analysis (i.e., Octrees and spatial reasoning), but does not require the design of tools (e.g., molds, dies) and so it can be used to produce shapes regardless of their complexity. The work presented in this paper makes use of the research work reported in [20] and [21] to obtain an integrated system able to automatically produce NNS parts of variable precision and complexity from multiple materials.

The rest of this paper is organized as follows. Section II introduces the RPNNS system and its main components, which are described in Section III to Section VII. The implementation of the system and the results obtained from the construction of two test components are presented in Section VIII. A discussion of the results is given in Section IX. Finally, the paper ends with conclusions and an overview of future research work.

\section{SYSTEM OVERVIEW}

An overview of the proposed RPNNS system is presented in Fig. 1. The system works by first subdividing a 3-D model into cubes of various sizes using an Octree decomposition approach. The Octree model is then "optimized" to reduce the number of cubes required to represent the model but without affecting the accuracy of the approximation. Following this, a stability analysis is carried out to detect unstable conditions that may affect the assembly of the Octree model. When unstable conditions are identified, the system adds supporting structures to eliminate the problems. After this stage the Octree model is suitable for physical fabrication and automated assembly planning is carried out. When the assembly plan has been completed, the system generates the instructions for the Octree assembly cell to construct the 
model and get an NNS component which can be postprocessed to obtain the net shape part.

\section{OCTREE DECOMPOSITION}

Octree representation is a hierarchical form of tessellation in which a volume occupied by a 3-D model is subdivided and approximated by cubes of varying sizes called 'octants'. Each of these cubes is identified by a locational code that represents its position in the Octree model. The relationships amongst octants can be viewed as a hierarchical tree structure where each branch is identified by the relative position of the octant in its parent node according to the six orientations R (right), L (left), $\mathrm{U}$ (up), D (down), F (front), and B (back). In this way octants can be identified as LDF, LDB, LUF, LUB, RDF, RDB, RUF, and RUB. The octants are also classified as full, empty or boundary, depending on their relative location in the 3-D model; inside, outside or partially inside. To create a maximal NNS component, both full and boundary octant are needed.

In this work, the Octree decomposition is exploited to approximate complex shapes with units of regular geometry and to create physical representations of models. Therefore, the size of octants must be limited to specific sizes according to the ability of the manufacturing process. For this reason, the traditional Octree decomposition cannot be used since the sizes and number of octants may not match those physically available. To ensure the Octree model matches the physical resources available for its construction, a new Octree decomposition method has been developed and is referred to as fixed-size-range decomposition [20]. In this approach, a 3-D model is approximated with cubes of certain range of sizes between a minimum value $s_{\min }$ and a maximum value $s_{\max }$. In the traditional Octree decomposition approach, the size of an octant can be computed as follows:

$$
s=\frac{a}{2^{l}}
$$

where $s$ is the size of octant, $a$ is the dimension of the bounding cube of the model, and $l$ is the level of decomposition. To control the size of octants, the fixed-size-range method computes a special value of the bounding cube based on the minimum size of octant required

$$
a_{c}=2^{l} \times s_{\min }
$$

where $a_{c}$ is the computed size of the bounding cube that will satisfy a minimum size of octant $s_{\min }$. Thus, the value of $a_{c}$ is computed by assuming different values of $l$ until $a_{c}$ becomes equal, or greater, than the original model's bounding cube $(a)$. Using this computed bounding cube the fixed-size-range algorithm performs the Octree decomposition until the maximum size of octant in the Octree model is smaller or equal to $s_{\max }$, and the minimum size of octant is greater or equal to $s_{\min }$.

The fixed-size-range decomposition approach guarantees that the sizes of octants will be inside the given range and, therefore, the physical representation of an Octree model can be done as octants will match the size ability of the manufacturing system. Fig. 2 presents the Octree decomposition of the ANC101 component using a size range of $[10,40] \mathrm{mm}$ which resulted in an Octree model comprised by 450 cubes of 10 and $20 \mathrm{~mm}$.

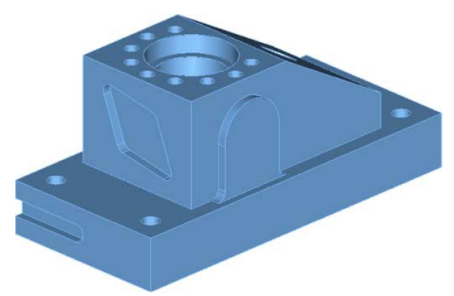

(a)

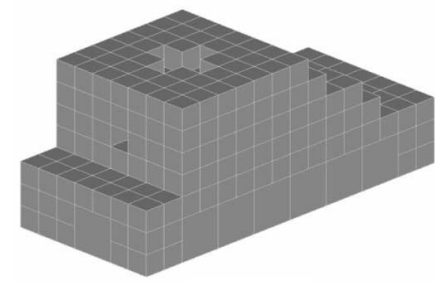

(b)
Fig. 2. Decomposition of the ANC101 component. (a) 3-D model. (b) Octree model.

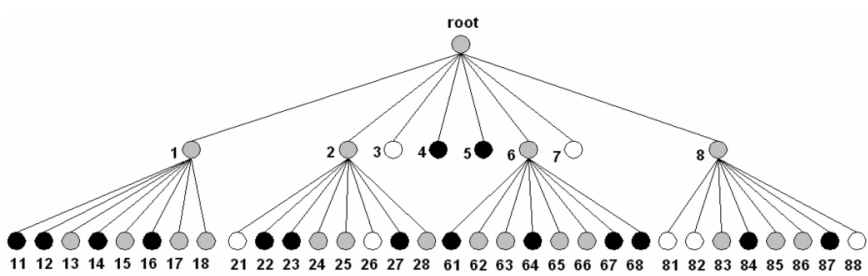

(a)

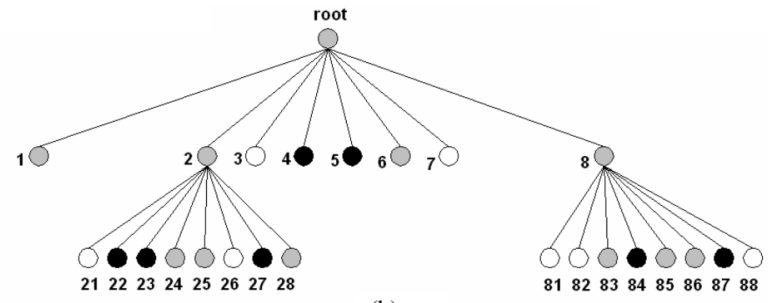

(b)

Fig. 3. Two-level Octree structure. (a) Before optimization. (b) After optimization.

\section{OCTREE OPTIMIZATION}

The process of reducing the number of cubes (i.e., octants) in an Octree model is referred to as the "Octree optimization." In this optimization process, the quality of the geometrical approximation must not decrease, so that the same Octree model can be obtained but with less number of cubes. Since no research work related to Octree optimization has been reported in the literature, a novel approach was developed to support the RPNNS system. This method is referred to as the Octree recomposition and is based on the reduction of octants by compressing the Octree structure [21]. The Octree recomposition algorithm uses a depth-first-search strategy [22], [23] to traverse the Octree structure and search for parent octants that have all their children contained in the Octree model (i.e., whose octant children are either boundary or full). When the algorithm finds one, it deletes the children and adds the parent to the Octree model. Since the Octree recomposition of octants may result in undesired large sizes of octants, a maximum size of octant is defined to control the optimization process. In this way, if the grouping of children results in an octant of larger size than the maximum size defined, then the reduction is not performed. This maximum size is defined according to the ability of the manufacturing system.

As an example, consider that an Octree decomposition of a 3-D model was completed until the second level of subdivision and the resultant Octree structure is as shown in Fig. 3(a), where black, gray, and white nodes represent full, boundary, 
and empty octants, respectively. Since the Octree decomposition was controlled to the second level of subdivision, all the full and boundary octants at this level were added to the Octree model to get a maximal NNS component. In this example, there are two groups of children that are either full or boundary (the children of parent 1 and the children of parent 6 ). These groups are replaced by their parents and the original Octree structure, which contained 41 octants, is reduced to the structure shown in Fig. 3(b), which contains just 25 octants and has the same approximation quality.

\section{StabiLITY ANALYSIS}

Unstable assembly conditions (e.g., overhanging or reentrant shapes) may cause binding problems that affect the accuracy and rigidity of the final component. The proposed solution to this stability problem in the RPNNS system is the use of "empty octants" (ignored in most of the Octree applications) as supporting structures, which will not be glued in place during the assembly of the model. In this way, empty octants could support the material above and be easily removed after the build. To support this approach, two algorithms for stability and part orientation analyses were developed and are explained in the following paragraphs.

The stability algorithm analyzes each octant in the Octree model to find its adjacent octants in the gravity direction. If adjacent octants exist, then the octant being analyzed is considered stable and does not require support. When no adjacent octants exist and the octant being analyzed is at the boundary of the model, the octant is considered as stable and no supports are required. However, when no adjacent octants exist and the octant being analyzed is not at the boundary, the octant is considered as unstable and the algorithm searches for adjacent empty octants in the gravity direction to add them to the Octree model as supporting cubes. An additional problem occurs when the size of the adjacent empty octant is bigger than the octant being analyzed. In this case, the algorithm subdivides the empty octant until an empty octant of the same or smaller size than the octant being analyzed is obtained. Since the addition of supporting octants may create new unstable conditions, the stability algorithm also analyzes the stability of the supporting octants being added. The traversing of the Octree structure to find adjacent octants is based on the neighbor finding method proposed in [24]. Fig. 4 illustrates an example where the stability analysis algorithm added a supporting cube and then an additional supporting cube to eliminate the unstable condition generated with the addition of the first support.

A part orientation algorithm to analyze different orientations of Octree models and determine the optimal orientation that results in the minimum number of supporting cubes, has been developed. Six orthogonal orientations parallel to the coordinate axes $(+\mathrm{X},+\mathrm{Y},+\mathrm{Z},-\mathrm{X},-\mathrm{Y},-\mathrm{Z})$ are considered in turn, and for each of these orientations the algorithm computes the number of supporting octants required. To analyze the orientations of an Octree model, the algorithm uses the stability algorithm considering different gravity directions.

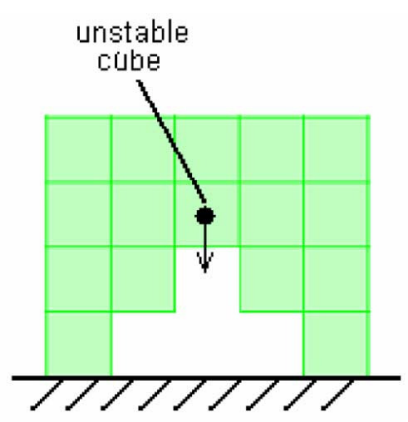

(a)

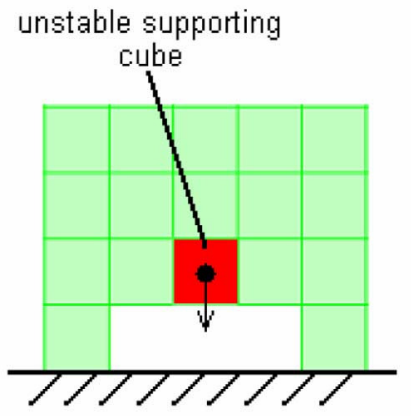

(c)

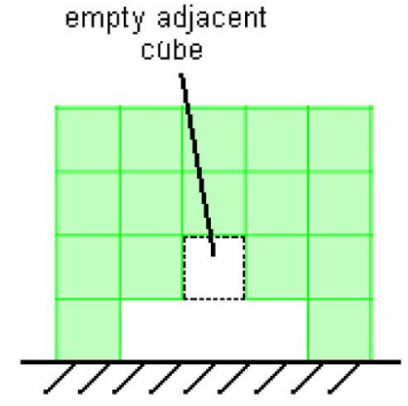

(b)

new

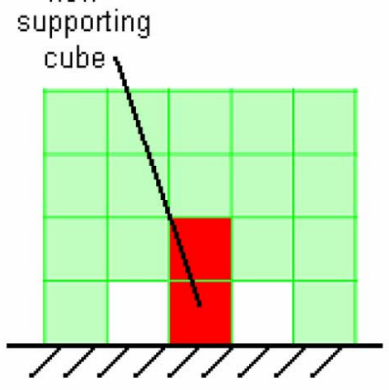

(d)
Fig. 4. Stability analysis. (a) Unstable cube. (b) Empty adjacent cube. (c) Supporting cube. (d) Additional supporting cube.

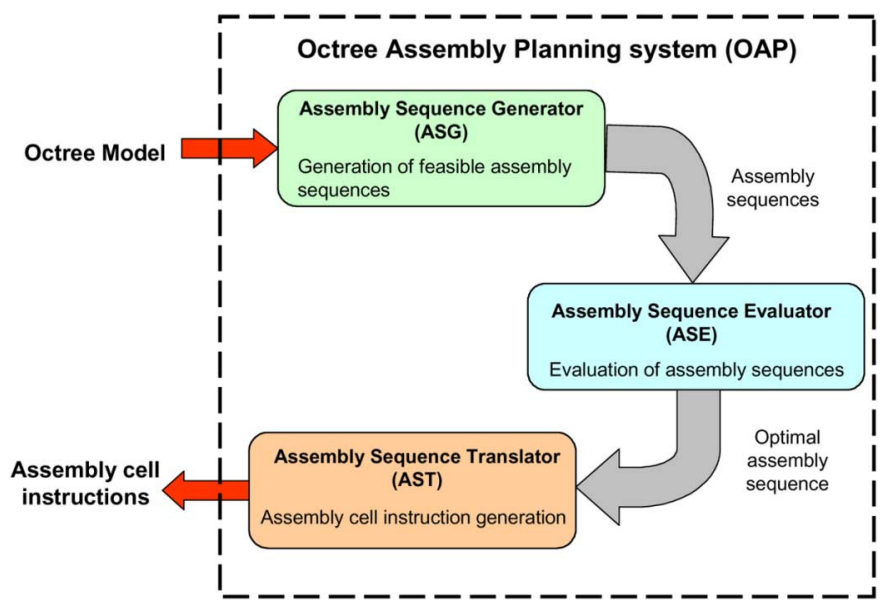

Fig. 5. Octree assembly planning system.

\section{Assembly PlanNING}

A complete assembly planning system for the construction of Octree models was developed to support the RPNNS system. This planning system, referred to as Octree assembly planning (OAP) system, comprises three main modules: 1) assembly sequence generator (ASG); 2) assembly sequence evaluator (ASE); and 3) assembly sequence translator (AST); see Fig. 5. The OAP system is based on a general assembly process defined by three moves, as shown in Fig. 6: move m1 takes a cube from the feeder to the binding system; move $\mathrm{m} 2$ takes a cube from the binding system to the boundary of the workspace; and move $\mathrm{m} 3$ takes a cube to its final assembly location. Within this framework, the assembly preferences are defined in terms of four variables: first axis (the progress assembly direction of 


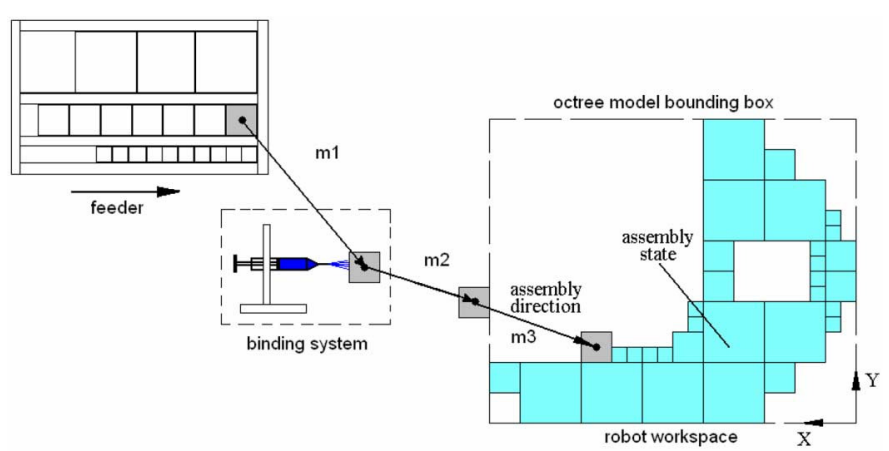

Fig. 6. General assembly process.

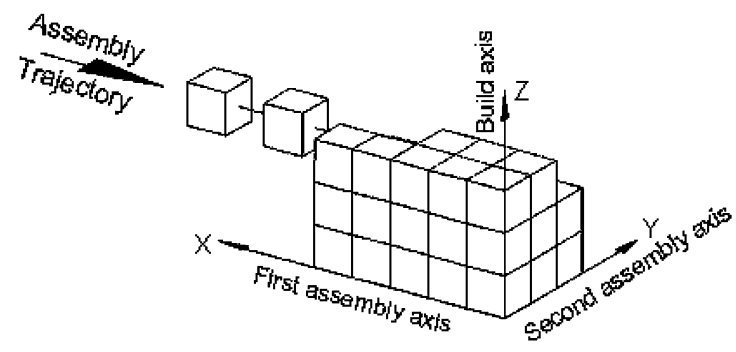

Fig. 7. Assembly variables.

cubes), second axis (the direction in which the first axis is incremented), build axis (normal direction to the first and second axis), and assembly trajectory (the approach direction used to assemble cubes). These assembly variables are illustrated in Fig. 7 and they have been limited to directions parallel to the coordinate system $(+\mathrm{X},+\mathrm{Y},+\mathrm{Z},-\mathrm{X},-\mathrm{Y},-\mathrm{Z})$.

\section{A. Assembly Sequence Generator (ASG)}

The ASG module generates feasible assembly sequences for an Octree model based on the following parameters: geometrical feasibility, mechanical feasibility, manipulability, accessibility, stability, assembly orientation, assembly progress preferences, and motion directions. From the analysis of these different criteria [21], 36 different sets of feasible assembly variables values were identified. Based on this, the ASG module uses the modular-assembly method presented in [20] to generate the 36 alternate assembly sequences. The modular-assembly method takes advantage of the spatial organization of the Octree structure to generate assembly sequences without performing any geometrical operation. Essentially, it is an ordered depth first search of the Octree structure that allows assembly sequence to be generated using a predefined collision free sequencing of each octant and its children. This order, referred to as the octants-assembly order, is defined by a set of values of the assembly variables and used to order the octants and generate the assembly sequence. As an example, consider the assembly of a level one Octree with first, second, build, and assembly trajectory variable values of $\mathrm{R}, \mathrm{B}, \mathrm{U}$, and $-\mathrm{B}$, respectively. The assembly order that satisfies these assembly preferences and guarantees a collision-free assembly is $\{\mathrm{LDF}, \mathrm{RDF}, \mathrm{LDB}, \mathrm{RDB}, \mathrm{LUF}, \mathrm{RUF}, \mathrm{LUB}, \mathrm{RUB}\}$, as shown in Fig. 8. The assembly of a level two Octree can be considered as a repetition of this sequence used to assemble the one level Octree. In this way, every octant in level one is con-
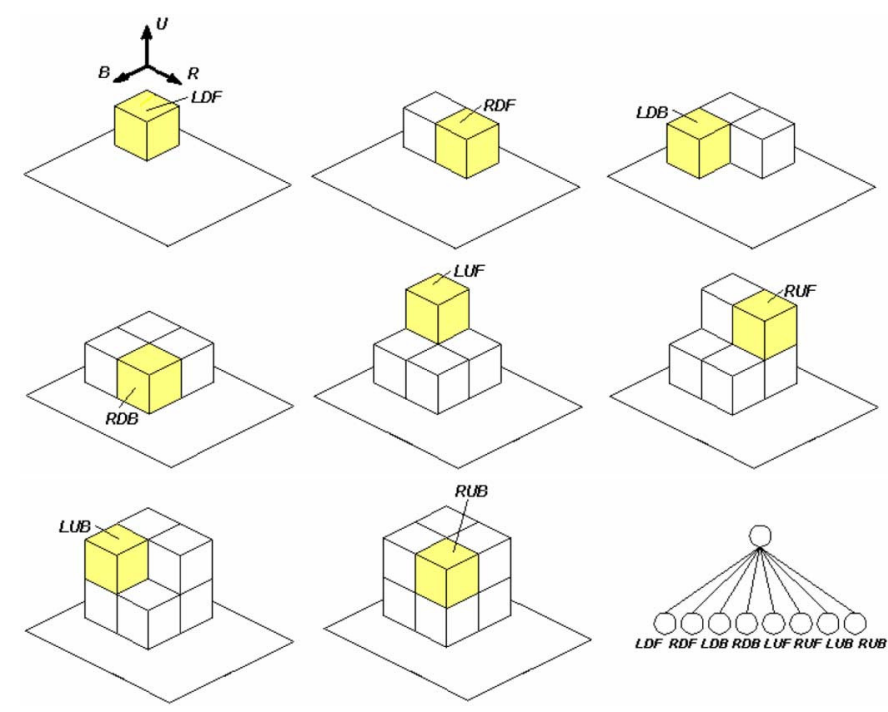

Fig. 8. Free-collision assembly order of a level one Octree.

sidered as being formed from recursive subassemblies ordered according to the octants-assembly order. By implementing an exhaustive, ordered, depth-first search of this form, an assembly sequence can then be generated.

\section{B. Assembly Sequence Evaluator (ASE)}

The aim of the ASE module is to find the optimal assembly sequence from the set of feasible assembly sequences generated previously. The evaluation of assembly sequences depends on criteria whose weightings vary according to each particular assembly process. In general, some of the most important criteria are: tool changes, orientation changes, fixture complexity, directionality, traveled distance, time, similar assembly operations, cost, and parallelism. Since in an Octree model all the parts have the same shape and are assembled using the same assembly tooling, the most significant criterion for the assembly sequence evaluation is the traveled distance, which is the length of the robot's assembly path to assemble an Octree model. According to the general assembly process defined in Fig. 6, the total traveled distance can be computed using the following equation:

$$
d_{T}=\sum_{i=1}^{n} d_{1 i}+d_{2 i}+d_{3 i}
$$

where $d_{T}$ is the total traveled distance, $n$ is the number of octants in the Octree model, and $d_{1}, d_{2}$, and $d_{3}$ are the traveled distances corresponding to the motions $\mathrm{m} 1, \mathrm{~m} 2$, and $\mathrm{m} 3$, respectively. These distances depend on the locations of the feeder, the binding system, and the assembly trajectory used to construct the model. Since the location of the feeder and the binding system are fixed and depend on the layout of the assembly cell, the traveled distance will vary only if the assembly trajectory varies. Based on this, the ASE module computes the traveled distance for each sequence and selects the optimal sequence that results in the minimum value.

\section{Assembly Sequence Translator (AST)}

The AST module is responsible in generating the instructions for the assembly cell to construct the Octree model based on 

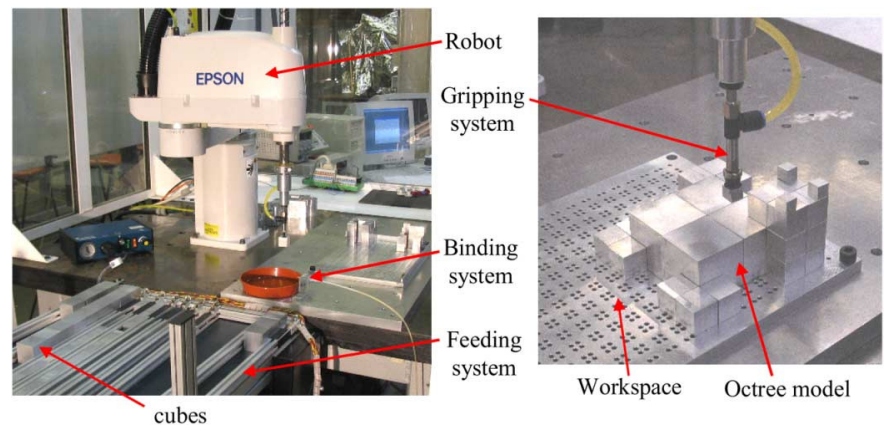

Fig. 9. Octree assembly cell.

the optimal assembly sequence found by the ASE module. An Epson ${ }^{\circledR}$ SCARA robot is used in the assembly cell to automatically construct Octree models and the COM interface to its programming language enables the use of Visual $\mathrm{C}++$ or any other software that supports ActiveX technology to run robotic applications.

\section{OCtReE ASSEMbly Cell}

The Octree assembly cell used in the RPNNS system is shown in Fig. 9. The main components of this cell are as follows.

\section{A. Robotic System}

An Epson ${ }^{\circledR}$ SCARA ES653S industrial robot (nominal repeatability of $\pm 0.02 \mathrm{~mm}$ ) and an Epson ${ }^{\circledR}$ SRC320 ABS multitask controller are used in the assembly cell. This robot controller has 16 inputs/ 16 outputs that can be easily read and generated via the SPEL API.

\section{B. Feeding System}

The feeding system stores and feeds the cubes in to the cell. A multisize cube feeder was designed and constructed. The feeder uses a belt conveyor to feed cubes through several lanes, which can be adjusted to different sizes by mean of separators. These separators can be added or removed to increase or decrease the number of lanes. The separators are adjusted in a tapered configuration to allow the cubes to be loaded more easily at the beginning of the lane and enable the automatic alignment of cubes at the end of the lane so the robot can pick them with accuracy. At the end of each lane, a sensor has been fitted to control the system, so whenever the robot removes a cube, the sensor detects it and runs the feeder until a new cube replaces the one that was taken.

\section{Binding System}

A simple but effective binding system consisting of a rinse bath of adhesive was adopted. Each cube being assembled is immersed into this bath before it is assembled. The advantage of this system is that the glue is applied to a cube in just one operation and therefore the rapidity of the system is improved. The disadvantage is that the glue is applied to all the faces of a cube even if they are not in contact. A cyanoacrylate-based adhesive was proposed because of its fast curing and high bond strength. The adhesive considered was Loctite ${ }^{\circledR} 426$ with a bond strength to aluminium of $2045 \mathrm{psi}$ and a curing speed of $5 \mathrm{~s}$ for a $40 \%$ of the full cured strength and a $60 \%$ of relative humidity [25].

\section{Gripping System}

The gripping system consists of a vacuum cup $(10 \mathrm{~mm}$ diameter) attached to the robot arm. This gripper uses the top face of a cube to grasp it but without obstructing the rest of the faces so they remain clear for glue application and assembly. The gripper applies the required pressure to hold the cubes in their positions during the assembly. The holding time is dependent on the type of the adhesive used.

\section{E. Workspace}

The current Octree assembly cell has a workspace area of $600 \mathrm{~mm} \times 300 \mathrm{~mm} \times 200 \mathrm{~mm}$. In this workspace, an aluminium base plate with holes to drain any excess of glue is located and a square support is also fitted at the origin of the workspace to provide alignment during the assembly.

\section{F. Raw Material}

Currently, aluminium solid cubes of 10, 20, and $40 \mathrm{~mm}$ are used in the system. These sizes of the cubes were selected based on the commercial sizes of aluminium square bars, the series of preferred numbers ISO R10 [26], and the dimension of the gripper. Some other materials such as wood, MDF, plastic, clay, CerroBend, etc., can also be used without requiring any significant change in the system.

\section{IMPLEMENTATION AND RESULTS}

The RPNNS application program has been developed in Visual $\mathrm{C}++$ and uses the ACIS $^{\circledR}$ geometric modeling kernel [27] to support the geometric operations required. The RPNNS system's user interface allows: solid model manipulation, Octree decomposition, Octree optimization, addition of supporting structures, automatic assembly planning, assembly sequence visualization and manual modification, assembly process setting, assembly operation testing, assembly instruction generation, and performance analysis.

Two components were used to test the RPNNS system. The first of them is the ex02 component shown in Fig. 10(a), which has a bounding box of $150 \mathrm{~mm} \times 100 \mathrm{~mm} \times 90 \mathrm{~mm}$. The Octree decomposition resulted in an Octree model with 567 cubes of 10,20 , and $40 \mathrm{~mm}$. The optimization of this Octree model was performed and the cubes were reduced from 567 to 378, i.e., a 33.3\% reduction [Fig. 10(b)]. The stability and orientation analysis of the model suggested the current orientation of the model as the optimal one requiring no supports. Multiple assembly sequences were then generated and evaluated. The results recommended an optimal assembly sequence with first, second, build and assembly trajectory variables of $\mathrm{X}, \mathrm{Y}, \mathrm{Z}$, and $-\mathrm{X}$, respectively. The computed traveled distance for this sequence was $247476 \mathrm{~mm}$. Using this optimal sequence, the model was constructed and the obtained NNS component is shown in Fig. 10(c). The assembly time for this model was $1 \mathrm{hr}, 1 \mathrm{~min}, 51 \mathrm{~s}$, and the total production time, from the Octree decomposition to the assembly of the model was $1 \mathrm{hr}, 21 \mathrm{~min}$, 


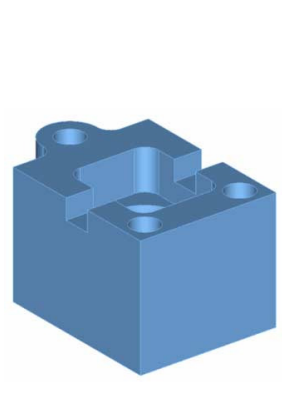

(a)

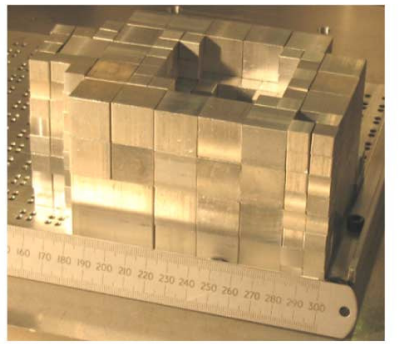

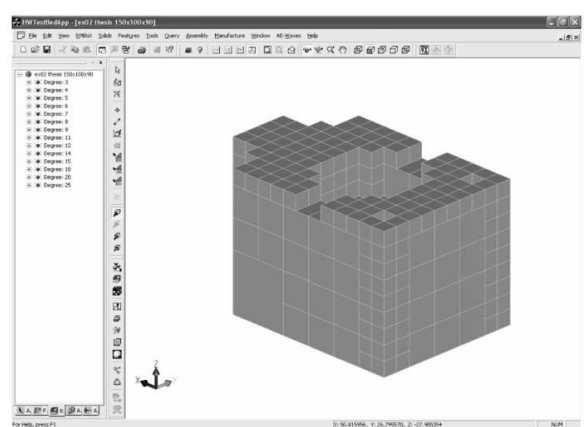

(b)

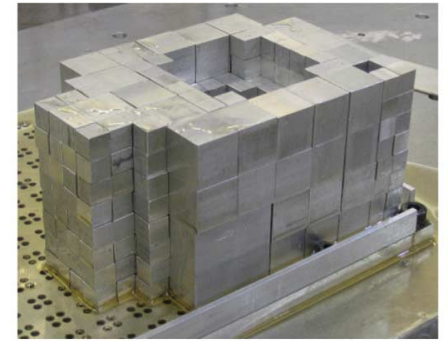

(c)

Fig. 10. Construction of the ex02 model. (a) CAD model. (b) Optimized Octree model. (c) NNS component.

$17 \mathrm{~s}$. The maximum misalignment error measured among cubes in the assembled component was $0.65 \mathrm{~mm}$.

The capability of the RPNNS system to construct complex shapes with overhangs and reentrants was tested with the truck model shown in Fig. 11(a), which has a bounding box of $144.8 \mathrm{~mm} \times 71.8 \mathrm{~mm} \times 71.9 \mathrm{~mm}$. The Octree decomposition resulted in an Octree model with 476 cubes of 10, 20, and $40 \mathrm{~mm}$ [Fig. 11(b)]. An Octree optimization of the model was carried out and the number of cubes was reduced from 476 to 203 , i.e., a $57.4 \%$ reduction [Fig. 11 (c)]. The stability and part orientation analysis of the optimized model led to the addition of 39 supports, as shown in Fig. 11(d). After this, the assembly planning of the model was performed and the results suggested an optimal assembly sequence with first, second, build and assembly trajectory variables of $\mathrm{X}, \mathrm{Y}, \mathrm{Z}$, and $-\mathrm{X}$, respectively, and with a traveled distance of $161286 \mathrm{~mm}$. The optimal sequence was then used to construct the final component [Fig. 11(e)]. The assembly time of the model was $39 \mathrm{~min}$ and the maximum misalignment error measured among cubes was $0.65 \mathrm{~mm}$. The total production time was $1 \mathrm{hr} 5 \mathrm{~min} 29 \mathrm{~s}$.

\section{DISCUSSION}

The implementation of the RPNNS system has been tested with the construction of two test components. The results showed that the construction of NNS components directly from CAD models using an Octree assembly-based approach is feasible. The quality of an NNS component produced in the RPNNS system is represented with the approximation quality coefficient $C_{\text {aq }}$ defined as the following nondimensional ratio [28]:

$$
C_{\mathrm{aq}}=\mathrm{RMV} / \mathrm{AMV}
$$

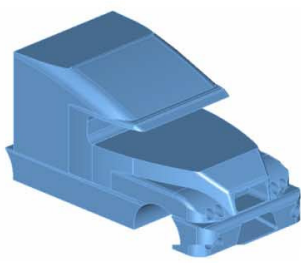

(a)

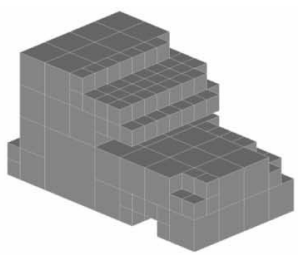

(c)

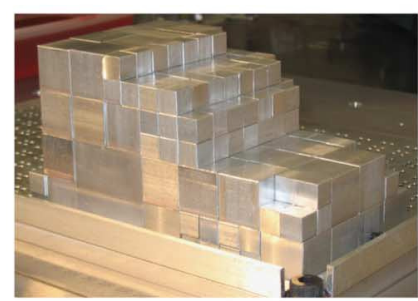

(e)

Fig. 11. Construction of the truck component. (a) CAD model. (b) Octree model. (c) Optimized Octree model. (d) Addition of supports. (e) NNS truck component.

where RMV is the volume of the actual model to be produced and AMV is the volume of the approximate model (NNS component). Since the actual model is always entirely inside the NNS component, AMV will be always greater or equal to RMV and, therefore, the value of $C_{\mathrm{aq}}$ will be always smaller or equal to 1 . A bigger value $C_{\mathrm{aq}}$ represents a better approximation of the component. In the case of the ex 02 and truck components, the obtained $C_{\text {aq }}$ was 0.847 and 0.678 , respectively. The best approximation was for the ex02 component because it contains big features and less complex shapes than the truck component. The analysis of several components has showed that the approximation quality depends on the inherent geometry of the model to be constructed and the range of cube sizes available in the Octree assembly cell. In general, large models will result in NNS components with good precision. Additionally, components of different precisions can be produced by controlling the range of cube sizes during the Octree decomposition.

The Octree optimization of the test components resulted in significant reductions of cubes. The greatest benefit was $57.4 \%$ for the truck model, which led to a proportional reduction in the assembly time. The results also showed that the execution time of the optimization is relatively short; for instance, the 
optimization of the truck component took 1 min $15 \mathrm{~s}$. In general, it is observed that the Octree optimization will always result in reductions of cubes and, consequently, in reductions of the assembly time. The stability and part orientation algorithms proved that empty octants can be used as supporting structures. These supporting cubes are not glued to the assembly, so they can be removed and reused once the assembly of the model has been completed. It is possible that some complex shapes may obstruct the removal of the supporting cubes. In these cases, the proposed solution is to define smaller sizes of supporting octants during the stability analysis. This will reduce the size of the supports and, therefore, the removal of them will be easier.

The assembly planning system was also tested with success and the results showed that the system is relatively fast. This is because the assembly planning algorithms exploit the Octree data structure and do not require any geometrical test. The assembly planning of the ex02 and truck components took 58.02 and $26.46 \mathrm{~s}$, respectively.

The capacity of the Octree assembly cell to construct Octree models was proved. The results suggested a general assembly unit time of 9.66 s/cube, i.e., the average time to assembly a cube in the Octree assembly cell is $9.66 \mathrm{~s}$. This assembly unit time can be used to estimate the construction time of Octree models when the number of cubes is known. The assembly cell is able to assemble cubes of different sizes and different materials. The main sources of dimensional errors in the Octree assembly cell are as follows.

- Tolerance in the dimension of cubes $( \pm 0.2 \mathrm{~mm})$;

- tolerance in the feeder lanes $(+0.2 \mathrm{~mm})$;

- flexibility of the gripper (deformation of the rubber);

- repeatability and accuracy of the robot $( \pm 0.02 \mathrm{~mm})$;

- layer thickness of adhesive (0.05 $\mathrm{mm}$ [25]).

The reduction of these sources of error would improve the accuracy of the RPNNS system.

The tolerance build up in assemblies can be predicted by two main methods: a) the worst case (WC) method and b) the root sum square (RSS) method. In a WC analysis, the assembly tolerance $\left(\mathrm{T}_{\mathrm{ASM}}\right)$ is determined by summing the component tolerances $\left(\mathrm{T}_{i}\right)$ linearly [29]. For one-dimensional assemblies, the assembly tolerance can be calculated according to the following expression:

$$
\mathrm{T}_{\mathrm{ASM}}=\sum \mathrm{T}_{i}
$$

In the case of the OcBlox system, the assembly tolerance can be estimated by considering the three dimensions separately as follows:

$$
\begin{aligned}
\mathrm{T}_{\mathrm{ASM} x} & =\sum \mathrm{T}_{i x} \\
\mathrm{~T}_{\mathrm{ASM} y} & =\sum \mathrm{T}_{j y} \\
\mathrm{~T}_{\mathrm{ASM} z} & =\sum \mathrm{T}_{k z}
\end{aligned}
$$

where $\mathrm{T}_{\mathrm{ASM} x}, \mathrm{~T}_{\mathrm{ASM} y}$, and $\mathrm{T}_{\mathrm{ASM} z}$ are the assembly tolerances in the directions $\mathrm{X}, \mathrm{Y}$, and $\mathrm{Z}$, respectively; and $\mathrm{T}_{i x}, \mathrm{~T}_{j y}$, and $\mathrm{T}$ $k z$ are the tolerances in the directions $\mathrm{X}, \mathrm{Y}$, and $\mathrm{Z}$ of the com-

\begin{tabular}{|c|c|c|c|c|}
\hline \multirow[t]{2}{*}{ Component } & \multirow{2}{*}{$\begin{array}{l}\text { No. } \\
\text { cubes } \\
\text { along } \\
\text { axis }\end{array}$} & \multicolumn{2}{|c|}{$\begin{array}{c}\text { Predicted } \\
\text { misalignment } \\
\text { T }_{\mathrm{ASM}}(\mathbf{m m})\end{array}$} & \multirow[t]{2}{*}{$\begin{array}{c}\text { Measured } \\
\text { misalignment } \\
(\mathrm{mm})\end{array}$} \\
\hline & & WC & RSS & \\
\hline Ex02 & 5 & 1.00 & 0.45 & 0.65 \\
\hline Truck & 7 & 1.40 & 0.53 & 0.65 \\
\hline
\end{tabular}
ponents along the directions $\mathrm{X}, \mathrm{Y}$, and $\mathrm{Z}$, respectively.
TABLE I

COMPARISON OF ASSEMBLY TOLERANCES

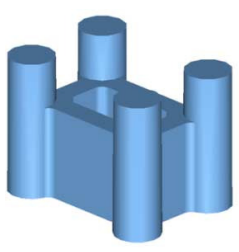

(a)

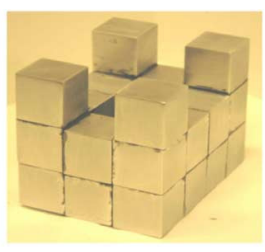

(b)

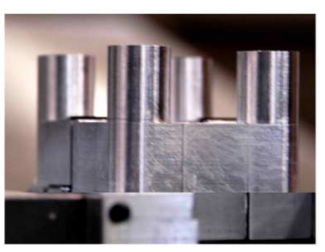

(c)
Fig. 12. CNC postprocessing of NNS components. (a) 3-D model. (b) NNS component. (c) Postprocessed by CNC.

In a RSS analysis, the assembly tolerance ( $\left.\mathrm{T}_{\mathrm{ASM}}\right)$ for one-dimensional assemblies can be calculated according to the following expression:

$$
\mathrm{T}_{\mathrm{ASM}}=\sqrt{\sum \mathrm{T}_{i}^{2}}
$$

Considering the three dimensions separately

$$
\begin{aligned}
\mathrm{T}_{\mathrm{ASM} x} & =\sqrt{\sum \mathrm{T}_{\mathrm{ix}^{2}}} \\
\mathrm{~T}_{\mathrm{ASM} y} & =\sqrt{\sum \mathrm{T}_{j y^{2}}} \\
\mathrm{~T}_{\mathrm{ASM} z} & =\sqrt{\sum \mathrm{T}_{k z^{2}}}
\end{aligned}
$$

Table I presents a comparison of the predicted values and the experimental values of the assembly tolerances of the two test components considering a $\mathrm{T}_{i}$ of $\pm 0.20 \mathrm{~mm}$. The results shows that the tolerances obtained in the assembly cell are smaller than the tolerances estimated using the WC analysis but are greater than the tolerances obtained using the RRS analysis.

To determine the feasibility of postprocessing by CNC machining the NNS components produced in the RPNNS system, several tests were carried out and the results suggested that the machining of glued assemblies of Octree models is possible, see Fig. 12. The benefit of producing this part by machining the NNS component rather than from a square stock was a reduction of $55.5 \%$ of the material removal. Postprocessing is required in all NNS processes but in the case of the RPNNS system, the traditional development cycle of NNS manufactured parts is reduced by eliminating the design and manufacture of production tooling (e.g., molds, dies, jigs, or fixtures). Currently the postprocessing of NNS components is done manually but the automatic generation of CNC code is under consideration.

\section{Conclusion}

A novel system for the rapid production of NNS components has been presented based on the Octree decomposition of CAD models which are automatically constructed using a "traditional" assembly cell. The system allows the automatic 
transfer of the 3-D model data generated by the Octree modeler, to an assembly planning system, which produces an optimal assembly sequence that, in turn, generates the instructions for the Octree assembly cell to construct NNS components. The RPNNS system is able to produce NNS components of different precisions and complexities, and has the advantage over other traditional NNS manufacturing processes in that it does not require the design and manufacture of production tooling, such as molds or dies.

The feasibility of the proposed RPNNS system was tested with the construction of two test components. The results showed that the construction of NNS components is feasible and that reductions in raw material usage and machining work can be obtained. The results also showed that the RPNNS system is best suited for large components because of the great benefits in terms of raw material usage, material removal, and production time that may be obtained. The system's limitations are accuracy, strength and overall build size, which can be improved by optimizing the system and reducing the sources of error.

Future work considers the automatic generation of CNC code for postprocessing NNS components. The use of different materials such as plastics, wood, MDF, clay, etc., is also considered as future work. The production of heterogeneous components will be also investigated. Several potential applications for assembly-based fabrication would require the RPNNS system to support cube construction at microscales or macroscales including the precision of manipulators and joining technologies. Additionally, assembly planner could be adapted to generate assembly precedence diagrams for cube-based fabrications, e.g., Soma puzzles or CAD assemblies that have been turned into Octrees [30].

\section{REFERENCES}

[1] P. Dahlman and M. Escursell, "High-pressure jet-assisted cooling: A new possibility for near net shape turning of decarburized steel," Int. J. Mach. Tools Manuf., vol. 44, pp. 109-115, 2004.

[2] Y. ZhouPing, D. Han, and X. YouLun, "Virtual prototyping of mold design: Geometric moldability analysis for near-net-shape manufactured parts by feature recognition and geometric reasoning," ComputerAided Des., vol. 33, pp. 137-154, 2001

[3] N. R. Chitkara and M. A. Bhutta, "Near net shape spline forging: An experimental investigation and simple upper bound analysis," Int. J. Mech. Sci., vol. 37, no. 12, pp. 1247-1268, 1995.

[4] N. R. Chitkara and M. A. Bhutta, "Near-net shape forging of spur gear forms: An analysis and some experiments," Int. J. Mech. Sci., vol. 38, no. 8-9, pp. 891-916, 1996.

[5] G. Hirt, R. Cremer, T. Witulski, and H. C. Tinius, "Lightweight near net shape components produced by thixoforming," Mater. Des., vol. 18, no. 4/6, pp. 315-321, 1997.

[6] N. R. Chitkara and Y. J. Kim, "Near-net shape forging of a crown gear: Some experimental results and an analysis," Int. J. Mach. Tools Manuf., vol. 41, pp. 325-346, 2001.

[7] A. G. Mamalis, A. Szalay, N. Gobl, I. Vajda, and B. Raveau, "Near netshape manufacturing of metal sheathed superconductors by high energy rate forming techniques," Mater. Sci. Eng., vol. B53, pp. 119-124, 1998.

[8] T. Sato, T. Besshi, and M. Matsui, "A new near net-shape forming process for alumina," J. Mater. Process. Technol., vol. 79, pp. 125-132, 1998.

[9] P. Greil, "Near net shape manufacturing of polymer derived ceramics," J. Eur. Ceramic Soc., vol. 18, pp. 1905-1914, 1998.

[10] R. Gilissen, J. P. Erauw, A. Smolders, E. Vanswijgenhoven, and J. Luyten, "Gelcasting, a near net shape technique," Mater. Des., vol. 21, pp. 251-257, 2000.
[11] C. Kaya and E. G. Butler, "Near net-shape manufacturing of alumina/ zirconia high temperature ceramics with fine scale aligned multiphase microstructures using co-extrusion," J. Mater. Process. Technol., vol. 135, pp. 137-143, 2003.

[12] P. Greil, "Near net shape manufacturing of ceramics," Mater. Chem. Phys., vol. 61, pp. 64-68, 1999.

[13] S. Das, M. Wohlert, J. J. Beaman, and D. L. Bourell, "Processing of titanium net shapes by SLS/HIP," Mater. Des., vol. 20, pp. 115-121, 1999.

[14] A. Devasenapathi, H. W. Ng, S. C. M. Yu, and A. B. Indra, "Forming near net shape freestanding components by plasma spraying," Mater. Lett., vol. 57, pp. 882-886, 2002.

[15] B. J. Mac Donald and M. S. J. Hashmi, "Near-net-shape manufacture of engineering components using bulge-forming processes: A review," J. Mater. Process. Technol., vol. 120, pp. 341-347, 2002.

[16] H. Hao, G. Zhang, J. P. Park, H. Y. Kim, and J. Z. Jin, "Twin-strand technology and microstructure analysis for the electromagnetic near net-shape casting of aluminium alloy," J. Mater. Process. Technol., vol. 142, pp. 526-531, 2003.

[17] X. Wu and J. Mei, "Near net shape manufacturing of components using direct laser fabrication technology," J. Mater. Process. Technol., vol. 135, pp. 266-270, 2003.

[18] P. L. Blackwell and A. Wisbey, "Laser-aided manufacturing technologies; Their application to the near-net shape forming of high-strength titanium alloy," J. Mater. Process. Technol., vol. 170, pp. 268-276, 2005.

[19] O. Owodunni, M. Salman, A. Mansor, and S. Hinduja, "Rapid development of product prototypes from reverse engineering and reconfigurable tooling," Time-Compression Technologies Mag., vol. 13, no. 4, pp. 45-49, Jul./Aug. 2005.

[20] H. Medellín, J. Corney, J. B. C. Davies, T. Lim, and J. M. Ritchie, "Algorithms for the physical rendering and assembly of Octree models," Comput. Aided-Design J., vol. 38, pp. 69-85, 2006.

[21] H. I. Medellín Castillo, "Assembly-based rapid production of near net shape components through octree decomposition of 3-D models," Ph.D. dissertation, Heriot-Watt Univ., Edinburgh, Scotland, U.K., 2005.

[22] ICS-270a: Notes 3: Lecture 3: State-Spaces and Uninformed Search. ICS 171, Fall 2004. Univ. California, Irvine, CA. [Online]. Available: http://www.ics.uci.edu/ dechter/ics-171/fall-04/lecture-notes/ 171_04-3-uninformed-search.ppt

[23] T. Cormen, C. Leiserson, and R. Rivest, Introduction to Algorithms. New York: McGraw-Hill, 1990.

[24] S. Hanan, "Neighbour finding in images represented by octrees," Computer Vision, Graphics and Image Processing, vol. 46, pp. 361-386, 1989.

[25] The design guide for bonding metals, Loctite ${ }^{\circledR}$, Henkel Technologies, vol. 4, 3/04. [Online]. Available: http://www.loctite.com

[26] ISO 3-1973, preferred numbers-Series of Preferred Numbers. ISO 17-1973, Guide to the Use of Preferred Numbers and Series of Preferred Numbers. ISO 497-1973, Guide to the Choice of Series of Preferred Numbers and of Series Containing More Rounded Values of Preferred Numbers.

[27] ACIS $^{\circledR}$ Geometric modeler, version 7.0. Spatial Technology Inc., Three Space Ltd., and Applied Geometry Corp., Boulder, CO. [Online]. Available: http://www.spatial.com

[28] H. Medellín, J. Corney, J. B. C. Davies, T. Lim, and J. M. Ritchie, "An automated system for the assembly of Octree models," Assembly Autom. J., vol. 24, no. 3, pp. 297-312, 2004, Emerald.

[29] "Manufacturing Review," ASME, vol. 1, no. 1, pp. 50-59, Mar. 1998.

[30] R. C. W. Sung, "Automatic assembly feature recognition and disassembly sequence generation," Ph.D. dissertation, Heriot-Watt Univ., Edinburgh, Scotland, U.K., 2001.

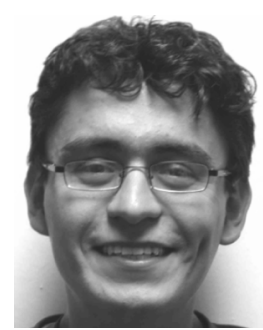

H. Medellín received the M.S. degree in mechanical engineering from the Universidad de Guanajuato, Mexico, and worked as a Design Engineer with the Comision Federal de Electricidad, Mexico, before receiving a Doctorate Scholarship, and the Ph.D. degree in the area of rapid prototyping and advanced manufacturing systems from Heriot-Watt University, Edinburgh, Scotland, U.K.

$\mathrm{He}$ is a Senior Lecturer in Mechanical Engineering at the Facultad de Ingeniería, Universidad Autonóma de San Luis Potosí, Mexico. His research interest includes $\mathrm{CAD} / \mathrm{CAM} / \mathrm{CAE}$ systems, rapid technologies, robotics, mechanisms, and biomechanics. 


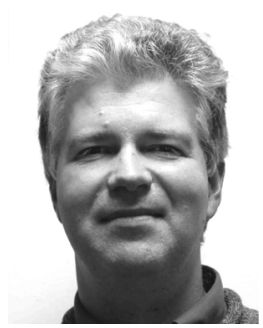

J. R. Corney is a Senior Lecturer in Mechanical Engineering at Heriot-Watt University, Edinburgh, Scotland, U.K., specializing in geometric modeling and has produced several exciting results in the field of geometric feature recognition, some of which have been developed for industrial applications. $\mathrm{He}$ is a member of the Digital Tools Manufacturing Group of the Scottish Manufacturing Institute and a member of the editorial board of the Journal of Computing and Information Science in Engineering. His current research includes rapid technologies and manufacturing systems, heterogeneous materials and modeling, and 3-D Internet search engines.

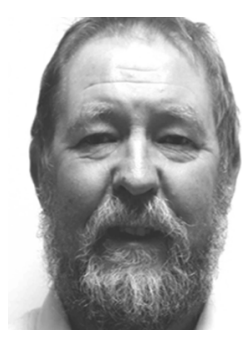

J. B. C. Davies is a Senior Lecturer in Mechanical Engineering at Heriot-Watt University, Edinburgh, Scotland, U.K. He has been active for nearly 15 years in the area of robotic handling and motion. Most recently, he has been the principal investigator on an EPSRC research project into the feasibility of using flexible actuators for underwater propulsion and material inspection. He is also a member of the editorial board of the Industrial Robot Journal and the Scottish Science Trust Public Understanding of Science Working Group.

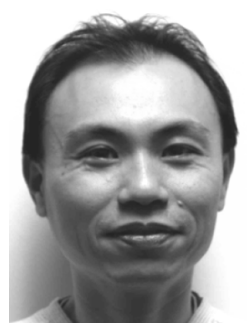

T. Lim is a Research Associate at Heriot-Watt University, Edinburgh, Scotland, U.K., specializing in geometric feature recognition and rapid manufacturing technologies. He is currently with the Department of Mechanical Engineering and is also active in the Department of Physics. His research interest includes biomaterials, CAD/CAM/CAE, computational geometry and analysis, virtual reality, medical engineering, and optics in computing.

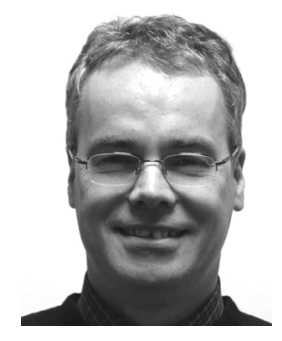

J. M. Ritchie is a Senior Lecturer in Mechanical Engineering at Heriot-Watt University, Edinburgh, Scotland, U.K., specializing in the areas of design, manufacturing, and manufacturing management. Recent research interests have included rapid prototyping, virtual reality applications in design and manufacturing, design process capability analysis, laser machining, and the application of Taguchi methods in the baking industry. He is also Academic Director of the University's Advanced Manufacturing Unit. 